\title{
Variant supercurrents and Noether procedure
}

\author{
Sergei M. Kuzenko ${ }^{\mathrm{a}}$ \\ School of Physics M013, The University of Western Australia, 35 Stirling Highway, 6009 Crawley W.A., Australia
}

Received: 30 September 2010 / Published online: 8 January 2011

(C) The Author(s) 2011. This article is published with open access at Springerlink.com

\begin{abstract}
Consistent supercurrent multiplets are naturally associated with linearized off-shell supergravity models. In S.M. Kuzenko, J. High Energy Phys. 1004, 022 (2010) we presented the hierarchy of such supercurrents which correspond to all the models for linearized $4 \mathrm{D} \mathcal{N}=1$ supergravity classified a few years ago. Here we analyze the correspondence between the most general supercurrent given in S.M. Kuzenko, J. High Energy Phys. 1004, 022 (2010) and the one obtained eight years ago in M. Magro et al., Ann. Phys. 298, 123 (2002) using the superfield Noether procedure. We apply the Noether procedure to the general $\mathcal{N}=1$ supersymmetric nonlinear sigma-model and show that it naturally leads to the so-called $\mathcal{S}$-multiplet, revitalized in Z. Komargodski, N. Seiberg, J. High Energy Phys. 1007, 017 (2010).
\end{abstract}

Inspired by a recent work of Komargodski and Seiberg [1], we have presented in [2] the hierarchy of supercurrent multiplets which are associated with the models for linearized $4 \mathrm{D} \mathcal{N}=1$ supergravity classified several years ago in [3]. The most general form of such a multiplet is as follows:

$$
\begin{aligned}
& \bar{D}^{\dot{\alpha}} J_{\alpha \dot{\alpha}}=\chi_{\alpha}+\mathrm{i} \eta_{\alpha}+D_{\alpha} X, \\
& \bar{D}_{\dot{\alpha}} \chi_{\alpha}=\bar{D}_{\dot{\alpha}} \eta_{\alpha}=\bar{D}_{\dot{\alpha}} X=0, \\
& D^{\alpha} \chi_{\alpha}-\bar{D}_{\dot{\alpha}} \bar{\chi}^{\dot{\alpha}}=D^{\alpha} \eta_{\alpha}-\bar{D}_{\dot{\alpha}} \bar{\eta}^{\dot{\alpha}}=0 .
\end{aligned}
$$

Here $J_{\alpha \dot{\alpha}}=\bar{J}_{\alpha \dot{\alpha}}$ denotes the supercurrent, while the chiral superfields $\chi_{\alpha}, \eta_{\alpha}$ and $X$ constitute the so-called multiplet of anomalies. The conservation law (1) incorporates six smaller supercurrent multiplets, of which three include $12+12$ operators (minimal supercurrents) and the rest describe $16+16$ components (reducible multiplets).

Let us recall the structure of the minimal supercurrent multiplets. The case

$$
\chi_{\alpha}=\eta_{\alpha}=0
$$

\footnotetext{
a e-mail: kuzenko@cyllene.uwa.edu.au
}

describes the famous Ferrara-Zumino multiplet [4]. It corresponds to the old minimal formulation for $\mathcal{N}=1$ supergravity [5-7]. Another choice,

$X=\eta_{\alpha}=0$,

corresponds to the new minimal supergravity $[8,9]$ (this supercurrent was studied in [10-12]). The third choice,

$X=\chi_{\alpha}=0$,

corresponds to the minimal $12+12$ supergravity formulation which was proposed a few years ago in [13]. Unlike the old minimal and the new minimal theories, this formulation is known at the linearized level only.

Among the three reducible supercurrents with $16+16$ components [2], the most interesting multiplet ${ }^{1}$ is singled out by the condition

$\eta_{\alpha}=0$.

It corresponds to the model (36) in [3], which can be shown to be a linearized version of the so-called $16+16$ supergravity $[14,15]$ known to be reducible [16]. After a 'death sentence' given to this multiplet in the late 1970s, it was recently resurrected by Komargodski and Seiberg [1]. These authors postulated the following supercurrent conservation law:

$$
\begin{aligned}
& \bar{D}^{\dot{\alpha}} J_{\alpha \dot{\alpha}}=\chi_{\alpha}+D_{\alpha} X, \quad \bar{D}_{\dot{\alpha}} \chi_{\alpha}=\bar{D}_{\dot{\alpha}} X=0, \\
& D^{\alpha} \chi_{\alpha}-\bar{D}_{\dot{\alpha}} \bar{\chi}^{\dot{\alpha}}=0
\end{aligned}
$$

and proved, using laborious component calculations, its consistency in the sense that $J_{\alpha \dot{\alpha}}$ contains a conserved energymomentum tensor and a conserved supersymmetry current.

\footnotetext{
${ }^{1}$ The other reducible supercurrents are obtained by setting either $\chi_{\alpha}=$ 0 or $X=0$. They appear to be less interesting than the one defined by (5), because the corresponding supergravity formulations are known at the linearized level only.
} 
The consistency of (6) is a built-in property within the approach of [2]. As argued in [1], the importance of the $\mathcal{S}$ multiplet (6) is that it exists for all known rigid supersymmetric theories, unlike the Ferrara-Zumino multiplet.

With regard to the most general supercurrent multiplet including $20+20$ operators, (1), it corresponds to a (twoparameter) sum of the three minimal $(12+12)$ linearized supergravity models ${ }^{2}$ listed in [3]. As shown in [3], such a theory is related to a linearized version of the non-minimal formulation for $\mathcal{N}=1$ supergravity $[17,18] .^{3}$

Let us represent the constrained chiral spinors $\chi_{\alpha}$ and $\eta_{\alpha}$ in (1) as vector-multiplet field strengths,

$$
\begin{aligned}
\chi_{\alpha}=-\frac{1}{4} \bar{D}^{2} D_{\alpha} V, & \bar{V}=V, \\
\eta_{\alpha}=-\frac{1}{4} \bar{D}^{2} D_{\alpha} U, & \bar{U}=U,
\end{aligned}
$$

associated with the prepotentials $V$ and $U$. Then, (1) turns into

$$
\begin{aligned}
& \bar{D}^{\dot{\alpha}} J_{\alpha \dot{\alpha}}=-\frac{1}{4} \bar{D}^{2} D_{\alpha}(V+\mathrm{i} U)+D_{\alpha} X, \\
& \bar{V}-V=\bar{U}-U=\bar{D}_{\dot{\alpha}} X=0 .
\end{aligned}
$$

This coincides with the general supercurrent derived eight years ago by Magro, Sachs and Wolf [19] with the aid of a modification of the superfield Noether procedure elaborated in [20] (see also [21]), provided the operators $V$ and $U$ are globally well-defined scalar superfields. However, for such operators $V$ and $U$, the supercurrent (8) proves to be equivalent to the Ferrara-Zumino one. Indeed, in this case we can introduce

$J_{\alpha \dot{\alpha}}^{(\mathrm{FZ})}:=J_{\alpha \dot{\alpha}}+\frac{1}{6}\left[D_{\alpha}, \bar{D}_{\dot{\alpha}}\right] V-\partial_{\alpha \dot{\alpha}} U$,

$X^{(\mathrm{FZ})}:=X-\frac{1}{12} \bar{D}^{2}(V+3 \mathrm{i} U)$,

where $J_{\alpha \dot{\alpha}}^{(\mathrm{I})}$ and $X^{(\mathrm{FZ})}$ obey the conservation equation

$\bar{D}^{\dot{\alpha}} J_{\alpha \dot{\alpha}}^{(\mathrm{FZ})}=D_{\alpha} X^{(\mathrm{FZ})}, \quad \bar{D}_{\dot{\alpha}} X^{(\mathrm{FZ})}=0$.

The obvious implication of this consideration (which appears to be implicit in the analysis of $[19,20]$ ) is that any rigid supersymmetric theory is characterized by a welldefined Ferrara-Zumino supercurrent.

\footnotetext{
${ }^{2}$ The gravitational superfield $H_{\alpha \dot{\alpha}}$ must be one and the same in all of these three models.

${ }^{3}$ The complex linear compensator $\Gamma$ of non-minimal supergravity, $\bar{D}^{2} \Gamma=0$, can be represented as $\Gamma=\sigma+G+\mathrm{i} F$, where $\sigma$ is a chiral scalar, $\bar{D}_{\dot{\alpha}} \sigma=0$, while $G$ and $F$ are real linear superfields, $\bar{D}^{2} G=G-\bar{G}=0$, and the same for $F$. The constrained superfields $\sigma, G$ and $F$ describe the compensators emerging in the three minimal supergravity formulations.
}

On the other hand, it has recently been argued in $[1,22]$ that there exist rigid supersymmetric field theories for which the Ferrara-Zumino multiplet is not well-defined. Such theories include (i) models with a Fayet-Iliopoulos term; and (ii) $\mathcal{N}=1$ nonlinear sigma-models with a non-exact Kähler form. Let us discuss the second example that appears to be most interesting. ${ }^{4}$ Consider the general $\mathcal{N}=1$ supersymmetric nonlinear sigma-model [25]

$S=\int \mathrm{d}^{8} z K\left(\Phi^{I}, \bar{\Phi}^{\bar{J}}\right)+\left\{\int \mathrm{d}^{6} z W\left(\Phi^{I}\right)+\right.$ c.c. $\}$.

The $\mathcal{S}$-multiplet, (6), for this model was found in [1]. It is

$J_{\alpha \dot{\alpha}}=\left(\bar{D}_{\dot{\alpha}} \bar{\Phi}^{\bar{J}}\right)\left(D_{\alpha} \Phi^{I}\right) K_{I \bar{J}}, \quad \chi_{\alpha}=-\frac{1}{2} \bar{D}^{2} D_{\alpha} K$,

$X=-2 W$,

and thus $V=2 K(\Phi, \bar{\Phi})$ and $U=0$. The three operators in (12) are clearly well-defined, in particular they are invariant under arbitrary Kähler transformations. However, since the Kähler potential $K$ is defined only locally in the target space, the operator $V$ is not globally well-defined in general. In particular, if the Kähler two-form of the target space is not exact, there is no way to define the operator $V$ globally. As a result, the improvement transformation (9) leads to ill-defined operators, and thus one is not allowed to use it.

We are going to demonstrate that the Noether procedure does not force both $V$ and $U$ to be globally well-defined operators. Let us apply a simple version of the Noether construction to the sigma-model (11). This model is superPoincare invariant. To start with, we replace the standard super-Poincaré transformation of $\Phi^{I}$ by a general local variation of the form:

$\delta \Phi^{I}=-\frac{1}{4} \bar{D}^{2}\left(L^{\alpha} D_{\alpha} \Phi^{I}\right)$,

where the parameter $L_{\alpha}(z)$ is an arbitrary spinor superfield. Such a variation can be recognized as a general coordinate transformation of a covariantly chiral scalar superfield in $\mathcal{N}=1$ supergravity (see textbooks [26, 27] for reviews). In the special case when $L_{\alpha}$ is chosen to correspond to a super-Poincaré transformation, the action does not change. If $L_{\alpha}$ is arbitrary, the variation of the action can be shown to be

$\delta S=\frac{1}{2} \int \mathrm{d}^{8} z L^{\alpha}\left\{\bar{D}^{\dot{\alpha}} J_{\alpha \dot{\alpha}}-\chi_{\alpha}-D_{\alpha} X\right\}+$ c.c.

\footnotetext{
${ }^{4}$ For theories with a Fayet-Iliopoulos term, the appropriate supercurrent is given by (3) [23, 24].
} 
where $J_{\alpha \dot{\alpha}}, \chi_{\alpha}$ and $X$ are defined in (12). If the equations of motion

$\frac{1}{4} \bar{D}^{2} K_{I}=W_{I}$

hold, the above variation vanishes, $\delta S=0$. Since the parameter $L^{\alpha}$ in (14) is arbitrary, we obtain the supercurrent conservation equation (6).

There are two important lessons that we can immediately learn from the above simple calculation. First of all, the Noether supercurrent for the sigma-model (11) coincides with the $\mathcal{S}$-multiplet. Secondly, the superfield Noether procedure is flexible enough in the sense that it does not force both prepotentials $V$ and $U$ in (8) to be globally well-defined operators.

The above sigma-model calculation is a streamlined version of the one described in [19]. Unfortunately, instead of giving the explicit expression for the $\mathcal{S}$-multiplet, the authors of [19] presented only the Ferrara-Zumino supercurrent of the sigma-model. Nevertheless, it is fair to say that the existence of the $\mathcal{S}$-multiplet as a consistent supercurrent multiplet is a by-product of the results obtained in $[19,20]$. It is due to the insight of Komargodski and Seiberg [1] that the physical significance of this multiplet has been uncovered.

Many rigid supersymmetric theories can be coupled to one of the existing off-shell versions of $\mathcal{N}=1$ supergravity. In such cases, the Noether procedure is not really necessary, for a consistent (rigid) supercurrent can be computed by varying the (curved-space) action with respect to the supergravity prepotentials, and then switching off the supergravity background. However, there exist theories for which (i) no coupling to supergravity is possible; or (ii) such a coupling is not known. For example, the sigma-model (11) cannot consistently be coupled to supergravity unless the target space is Hodge-Kähler [28]. On the other hand, it is not known how to couple supergravity to the off-shell gauge models for higher-spin massless multiplets [29, 30] (see [27] for a review). In all such cases, the Noether procedure $[19,20]$ becomes indispensable.

It was conjectured in [1] that the $\mathcal{S}$-multiplet, (6), exists for all rigid supersymmetric theories. On the other hand, both the linearized supergravity analysis and the Noether procedure allow for the more general conservation equation (1). It would be interesting to understand whether it is always possible, modulo an improvement transformation, to set $\eta_{\alpha}=0$ or not.

Acknowledgements The author is grateful to Ivo Sachs for reminding him of [19]. This work is supported in part by the Australian Research Council.

Open Access This article is distributed under the terms of the Creative Commons Attribution Noncommercial License which permits any noncommercial use, distribution, and reproduction in any medium, provided the original author(s) and source are credited.

\section{References}

1. Z. Komargodski, N. Seiberg, Comments on supercurrent multiplets, supersymmetric field theories and supergravity. J. High Energy Phys. 1007, 017 (2010). arXiv:1002.2228 [hep-th]

2. S.M. Kuzenko, Variant supercurrent multiplets. J. High Energy Phys. 1004, 022 (2010). arXiv:1002.4932 [hep-th]

3. S.J. Gates Jr., S.M. Kuzenko, J. Phillips, The off-shell $(3 / 2,2)$ supermultiplets revisited. Phys. Lett. B 576, 97 (2003). arXiv:hep-th/0306288

4. S. Ferrara, B. Zumino, Transformation properties of the supercurrent. Nucl. Phys. B 87, 207 (1975)

5. J. Wess, B. Zumino, Superfield Lagrangian for supergravity. Phys. Lett. B 74, 51 (1978)

6. K.S. Stelle, P.C. West, Minimal auxiliary fields for supergravity. Phys. Lett. B 74, 330 (1978)

7. S. Ferrara, P. van Nieuwenhuizen, The auxiliary fields of supergravity. Phys. Lett. B 74, 333 (1978)

8. V.P. Akulov, D.V. Volkov, V.A. Soroka, Generally covariant theories of gauge fields on superspace. Theor. Math. Phys. 31, 285 (1977)

9. M.F. Sohnius, P.C. West, An alternative minimal off-shell version of $N=1$ supergravity. Phys. Lett. B 105, 353 (1981)

10. S.P. Bedding, W. Lang, Linearized superfield formulation of the new minimal $N=1$ supergravity. Nucl. Phys. B 196, 532 (1982)

11. P.S. Howe, K.S. Stelle, P.K. Townsend, The vanishing volume of $N=1$ superspace. Phys. Lett. B 107, 420 (1981)

12. S.J. Gates Jr., M. Roček, W. Siegel, Solution to constraints for $n=0$ supergravity. Nucl. Phys. B 198, 113 (1982)

13. I.L. Buchbinder, S.J. Gates Jr., W.D. Linch, J. Phillips, New 4D, $N=1$ superfield theory: Model of free massive superspin-3/2 multiplet. Phys. Lett. B 535, 280 (2002). arXiv:hep-th/0201096

14. G. Girardi, R. Grimm, M. Müller, J. Wess, Antisymmetric tensor gauge potential in curved superspace and a $(16+16)$ supergravity multiplet. Phys. Lett. B 147, 81 (1984)

15. W. Lang, J. Louis, B.A. Ovrut, $(16+16)$ supergravity coupled to matter: The low-energy limit of the superstring. Phys. Lett. B 158, 40 (1985)

16. W. Siegel, 16/16 supergravity. Class. Quantum Gravity 3, L47 (1986)

17. P. Breitenlohner, Some invariant Lagrangians for local supersymmetry. Nucl. Phys. B 124, 500 (1977)

18. W. Siegel, S.J. Gates Jr., Superfield supergravity. Nucl. Phys. B 147, 77 (1979)

19. M. Magro, I. Sachs, S. Wolf, Superfield Noether procedure. Ann. Phys. 298, 123 (2002). arXiv:hep-th/0110131

20. H. Osborn, $N=1$ superconformal symmetry in fourdimensional quantum field theory. Ann. Phys. 272, 243 (1999). arXiv:hep-th/9808041

21. K.-I. Shizuya, Supercurrents and superconformal symmetry. Phys. Rev. D 35, 1848 (1987)

22. Z. Komargodski, N. Seiberg, Comments on the Fayet-Iliopoulos term in field theory and supergravity. J. High Energy Phys. 0906, 007 (2009). arXiv:0904.1159 [hep-th]

23. K.R. Dienes, B. Thomas, On the inconsistency of FayetIliopoulos terms in supergravity theories. Phys. Rev. D 81, 065023 (2010)

24. S.M. Kuzenko, The Fayet-Iliopoulos term and nonlinear selfduality. Phys. Rev. D 81, 085036 (2010). arXiv:0911.5190 [hepth]

25. B. Zumino, Supersymmetry and Kähler manifolds. Phys. Lett. B 87, 203 (1979)

26. S.J. Gates Jr., M.T. Grisaru, M. Roček, W. Siegel, Superspace, or One Thousand and One Lessons in Supersymmetry (Benjamin/Cummings, Reading, MA, 1983). hep-th/0108200 
27. I.L. Buchbinder, S.M. Kuzenko, Ideas and Methods of Supersymmetry and Supergravity or a Walk Through Superspace (IOP, Bristol, 1998)

28. E. Witten, J. Bagger, Quantization of Newton's constant in certain supergravity theories. Phys. Lett. B 115, 202 (1982)
29. S.M. Kuzenko, A.G. Sibiryakov, V.V. Postnikov, Massless gauge superfields of higher half integer superspins. JETP Lett. 57, 534 (1993) [Pis'ma Zh. Eksp. Teor. Fiz. 57 (1993) 521]

30. S.M. Kuzenko, A.G. Sibiryakov, Massless gauge superfields of higher integer superspins. JETP Lett. 57, 539 (1993) [Pis'ma Zh. Eksp. Teor. Fiz. 57, 526 (1993)] 